\title{
Smooth negligibility of compact sets in infinite-dimensional Banach spaces, with applications
}

\author{
D. Azagra ${ }^{1}$, T. Dobrowolski ${ }^{2}$ \\ 1 Departamento de Análisis Matemático, Facultad de Ciencias Matemáticas, Universidad \\ Complutense, E-28040, Madrid, Spain (e-mail: daniel@sunam1.mat.ucm.es) \\ 2 Department of Mathematics, Pittsburg State University, Pittsburg, KA 66762, USA \\ (e-mail: tdobrowo@mail.pittstate.edu)
}

Received: 25 November 1997 / Revised version: 2 April 1998

Mathematics Subject Classification (1991): 46B20, 58B99

\section{Introduction}

To the best of the authors' knowledge, what one could call negligibility theory in infinite-dimensional Banach spaces started in 1953 when Victor L. Klee [22] proved that, if $X$ is either a non-reflexive Banach space or an infinite-dimensional $L^{p}$ space and $K$ is a compact subset of $X$, there exists a homeomorphism between $X$ and $X \backslash K$ which is the identity outside a neighborhood of $K$. Klee also proved that for those infinite-dimensional Banach spaces $\mathrm{X}$ the unit sphere is homeomorphic to any of the closed hyperplanes in $\mathrm{X}$, and gave a topological classification of convex bodies in Hilbert spaces. In 1966, C. Bessaga [2] proved that every infinite-dimensional Hilbert space is $C^{\infty}$ diffeomorphic to its unit sphere. He had previously used his so-called non-complete norm technique to simplify those results of Klee's and to generalize them to the class of all Banach spaces.

The real-analytic and smooth negligibility of compact sets in Banach spaces was studied by the second-named author [13], who developed Bessaga's non-complete norm technique in the smooth case and generalized some of the results of [2]. He [13] showed that for every infinite-dimensional Banach space $X$ having a $C^{p}$ non-complete norm $\omega$, and for every compact set $K$ in $X$, the space $X$ is $C^{p}$ diffeomorphic to $X \backslash K$. If, in addition, $X$ has an equivalent $C^{p}$ smooth norm $\|\cdot\|$ then one can deduce that the sphere $S=\{x \in X \mid\|x\|=1\}$ is $C^{p}$ diffeomorphic to any of the hyperplanes in $X$. He also used his results on smooth negligibility to give a 
classification of smooth convex bodies in WCG Banach spaces (see [15]). Concerning $C^{p}$ smooth negligibility of compact sets, the largest class of Banach spaces within which these results were known to be true was that of all Banach spaces which can be linearly injected into some $c_{0}(\Gamma)$. It should be remarked that there are examples of spaces with $C^{\infty}$ smooth equivalent norms which do not linearly embed into any $c_{0}(\Gamma)$. An example of such a (nonseparable) Banach space is given in ([11], Ex. VI.8.8), and it may be chosen to be $C(K)$ for a certain compact set $K$. So, when one wants to generalize those results to every infinite-dimensional Banach space having a $C^{p}$ smooth norm, one faces the following problem: Does every infinitedimensional Banach space with a $C^{p}$ smooth equivalent norm admit a $C^{p}$ smooth non-complete norm too? This intriguing question remains unsolved.

Without proving the existence of smooth non-complete norms, the firstnamed author has recently shown [1] that every Banach space $(X,\|\cdot\|)$ with a (not necessarily equivalent) $C^{p}$ smooth norm $\varrho$ is $C^{p}$ diffeomorphic to $X \backslash\{0\}$ and, moreover, that every hyperplane $H$ in $X$ is $C^{p}$ diffeomorphic to the sphere $\{x \in X \mid \varrho(x)=1\}$. In this paper, we strengthen the asymmetric norm technique of deleting points introduced in [1] so as to generalize some results on smooth negligibility of compacta and subspaces to the class of all Banach spaces having a (not necessarily equivalent) $C^{p}$ smooth norm. We also give a full smooth classification of the convex bodies of every Banach space. In particular, we show that every smooth convex body containing no linear subspaces in an infinite-dimensional Banach space is diffeomorphic to a half-space.

These results enable us to enlarge the class of spaces for which some other applications of negligibility are valid. At the end of the paper we give a sample of such applications which includes Garay's theorems [16, 17] concerning the existence of solutions to ordinary differential equations and cross-sections of solution funnels in Banach spaces, as well as sharper statements of Klee's results [22] on periodic homeomorphisms without fixed points.

\section{Removing compact sets from a Banach space}

In this section we will give a method of removing compacta smoothly from an infinite-dimensional Banach space having a (not necessarily equivalent) smooth norm. Let us state our main result.

Theorem 2.1. Let $(X,\|\cdot\|)$ be an infinite-dimensional Banach space with a (not necessarily equivalent) $C^{p}$ smooth norm $\varrho$. Then, for every compact set $K \subset X$, there exists a $C^{p}$ diffeomorphism $\varphi$ between $X$ and $X \backslash K$. Moreover, for each open $\varrho$-ball $B$ containing $K$, we can additionally require that $\varphi$ be the identity outside $B$. 
The proof of this result is quite long and technical. In what follows, we state and prove a number of auxiliary results that we will use in the proof of 2.1. We will show that a mapping $\psi(x)=x+p(f(x)), x \in X \backslash K$, for certain $f: X \rightarrow[0, \infty)$ with $f^{-1}(K)=0$ and $p:(0, \infty) \rightarrow X$, establishes a $C^{p}$ diffeomorphism between $X \backslash K$ and $X$. The map $\psi$ can be viewed as a "small" perturbation of the identity map, and its bijectivity is obtained by an application of Lemma 2.2, a simple fixed point fact whose proof is omitted. In order that the perturbation $p \circ f$ be small, $p$ and $f$ must satisfy some Lipschitzian-type conditions with respect to a certain assymetric distance induced by an assymetric norm $\omega$. Such a norm is constructed in Lemma 2.3. Lemma 2.5 gives us a required path $p$ which "avoids" compact sets; this, in turn, will make $K$ "disappear". Lemma 2.6 provides us with a required function $f(x)$ which can be viewed as a smooth substitute for the asymmetric $\omega$-distance function from $x$ to $K$. The $C^{p}$ diffeomorphism $\psi$ is constructed in such a way that it is the identity outside a certain $\omega$-ball. Finally, in order to obtain a required diffeomorphism $\varphi$, we make use of Lemma 2.7, which yields a radial $C^{p}$ diffeomorphism of $X$ sending a $\varrho$-ball onto an $\omega$-ball. This is the strategy of our proof of 2.1.

Lemma 2.2. Let $F:(0, \infty) \longrightarrow[0, \infty)$ be a continuous function such that, for every $\beta \geq \alpha>0, F(\beta)-F(\alpha) \leq \frac{1}{2}(\beta-\alpha)$ and $\limsup _{t \rightarrow 0^{+}} F(t)>0$. Then there exists a unique $\alpha>0$ such that $F(\alpha)=\alpha$.

The following lemma shows that for every Banach space with a $C^{p}$ smooth norm there exists a functional which will act as a smooth noncomplete norm in its absence.

Lemma 2.3. Let $(X,\|\cdot\|)$ be an infinite-dimensional Banach space having a (not necessarily equivalent) $C^{p}$ smooth norm $\varrho$. Then there exists a continuous functional $\omega: X \longrightarrow[0, \infty)$ which is $C^{p}$ smooth on $X \backslash\{0\}$ and satisfies the following conditions:

1. $\omega(x+y) \leq \omega(x)+\omega(y)$ and, consequently, $\omega(x)-\omega(y) \leq \omega(x-y)$, for every $x, y \in X$;

2. $\omega(r x)=r \omega(x)$ for every $x \in X$ and $r \geq 0$;

3. $\omega(x)=0$ if and only if $x=0$;

4. $\omega\left(\sum_{k=1}^{\infty} z_{k}\right) \leq \sum_{k=1}^{\infty} \omega\left(z_{k}\right)$ for every convergent series $\sum_{k=1}^{\infty} z_{k}$; and

5. For every $\delta>0$, there exists a sequence of linearly independent vectors $\left(y_{k}\right)$ satisfying

$$
\omega\left(y_{k}\right) \leq \frac{\delta}{4^{k+1}}
$$

for every $k \in \mathbb{N}$, and with the property that for every compact set $K \subset X$ there exists $n_{0} \in \mathbb{N}$ such that

$$
\inf \left\{\omega\left(z-\sum_{k=1}^{n} y_{k}\right) \mid n \geq n_{0}, z \in K\right\}>0
$$


Remark 2.4. The functional $\omega$ is not necessarily a norm; in general, $\omega(x) \neq$ $\omega(-x)$. However, for our purposes, we will refer to $\omega$ as to an assymetric non-complete norm.

Proof of lemma 2.3. We will consider three cases.

Case I: The norm $\varrho$ is complete and the space $X$ is non-reflexive.

The norm $\varrho$ is continuous with respect to $\|\cdot\|$ (because it is $C^{p}$ smooth), and complete. Hence, according to the open mapping theorem, $\varrho$ is a $C^{p}$ smooth equivalent norm in $X$, and we can assume that $\varrho=\|\cdot\|$. Since $X$ is not reflexive, according to James's theorem [21], there exists a continuous linear functional $T: X \longrightarrow \mathbb{R}$ such that $T$ does not attain its norm. We may assume $\|T\|=1$, so that $\sup \{T(x):\|x\|=1\}=1$, and yet $T(x)<\|x\|$ for every $x \neq 0$. Let us define $\omega: X \longrightarrow[0, \infty)$ by

$$
\omega(x)=\|x\|-T(x) .
$$

Note that $\omega(x)=0$ if and only if $x=0, \omega(x+y) \leq \omega(x)+\omega(y)$ for every $x, y \in X$, and $\omega(r x)=r \omega(x)$ for each $r>0$, although $\omega$ is not a norm in $X$ because $\omega(x) \neq \omega(-x)$ in general. The property $\omega(z+y) \leq \omega(z)+\omega(y)$ implies that $\omega(x)-\omega(y) \leq \omega(x-y)$, as well as $\omega\left(\sum_{k=1}^{\infty} z_{k}\right) \leq \sum_{k=1}^{\infty} \omega\left(z_{k}\right)$ for every convergent series $\sum_{k=1}^{\infty} z_{k}$. Then $\omega$ satisfies properties $1-4$, and it only remains to check that $\omega$ satisfies property 5 . For a given $\delta>0$, since $\sup \{T(x):\|x\|=1\}=1$, there exists a sequence $\left(y_{k}\right)$ such that $\left\|y_{k}\right\|=1$ and $\omega\left(y_{k}\right)=\left\|y_{k}\right\|-T\left(y_{k}\right) \leq \frac{\delta}{4^{k+1}}$ for every $k \in \mathbb{N}$, that is, $\omega$ satisfies the first part of property 5 . Clearly, we may assume that the vectors $\left(y_{k}\right)$ are linearly independent. We only have to check that for such a sequence $\left(y_{k}\right)$ the following condition is also satisfied: for every compact set $K \subset X$ there exists $n_{0}=n_{0}(K) \in \mathbb{N}$ such that

$$
\inf \left\{\omega\left(z-\sum_{k=1}^{n} y_{k}\right) \mid n \geq n_{0}, z \in K\right\}>0 .
$$

So, let $K$ be a compact set, let $M>0$, and take $R>0$ such that $\|z\| \leq R$ for every $z \in K$. Since $T\left(y_{k}\right) \rightarrow 1$ as $k \rightarrow \infty$, we can find $n_{0} \in \mathbb{N}$ such that $\sum_{k=1}^{n} T\left(y_{k}\right)>M+R$ for every $n \geq n_{0}$. Then we have

$$
\begin{aligned}
& \omega\left(z-\sum_{k=1}^{n} y_{k}\right)=\left\|z-\sum_{k=1}^{n} y_{k}\right\|-T\left(z-\sum_{k=1}^{n} y_{k}\right) \geq-T\left(z-\sum_{k=1}^{n} y_{k}\right) \\
& =-T(z)+T\left(\sum_{k=1}^{n} y_{k}\right) \geq-\|z\|+T\left(\sum_{k=1}^{n} y_{k}\right)=-\|z\|+\sum_{k=1}^{n} T\left(y_{k}\right) \\
& \geq-R+M+R=M
\end{aligned}
$$


whenever $n \geq n_{0}, z \in K$. This proves that

$$
\inf \left\{\omega\left(z-\sum_{k=1}^{n} y_{k}\right) \mid n \geq n_{0}, z \in K\right\} \geq M>0 .
$$

Case II: The norm $\varrho$ is non-complete.

Define $\omega=\varrho$. As $\varrho$ is a $C^{p}$ smooth norm, it is clear that $\omega$ satisfies conditions 1-4. Let us see that $\omega$ also satisfies condition 5. Since the norm $\omega$ is non-complete, for every $\delta>0$ we can find a sequence $\left(y_{k}\right)$ in $X$ such that $\omega\left(y_{k}\right) \leq \frac{\delta}{4^{k+1}}$ for each $k$, and a point $\hat{y}$ in the completion of $(X, \omega)$, denoted by $(\hat{X}, \hat{\omega})$, such that $\hat{y} \notin X$, and $\lim _{n \rightarrow \infty} \hat{\omega}\left(\hat{y}-\sum_{k=1}^{n} y_{k}\right)=0$. So the first part of property 5 is satisfied. Moreover it is clear that the sequence $\left(y_{k}\right)$ can be chosen in such a way that $\left\{y_{k} \mid k=1,2, \ldots\right\}$ is a linearly independent set of vectors. It only remains to check that for such a sequence $\left(y_{k}\right) \subset X$ and for every compact set $K \subset X$ there exists $n_{0} \in \mathbb{N}$ such that

$$
\inf \left\{\omega\left(z-\sum_{k=1}^{n} y_{k}\right) \mid n \geq n_{0}, z \in K\right\}>0 .
$$

Let $K$ be a compact set of $(X,\|\cdot\|)$. It is clear that $\hat{\omega}\left(z-\sum_{k=1}^{n} y_{k}\right)$ converges to $\hat{\omega}(z-\hat{y})$ uniformly on $z \in K \subset(\hat{X}, \hat{\omega})$. Since $K \subset(\hat{X}, \hat{\omega})$ is compact, $\hat{\omega}(z-\hat{y})$ is bounded away from zero for all $z \in K$. Consequently, we get our estimate.

Case III: The norm $\varrho$ is complete and the space $X$ is reflexive.

This case can be reduced to the previous one by showing that every infinite-dimensional reflexive space has a non-complete $C^{\infty}$ smooth norm $\omega$. Indeed, for every reflexive space $X$ there exists a linear injection $J$ : $X \longrightarrow c_{0}(\Gamma)$ for some (infinite) set $\Gamma$ (see, e.g., [11], chapter VI, p. 246). It is also well known that for an infinite set $\Gamma$, the space $c_{0}(\Gamma)$ is $c_{0}$-saturated, that is, every infinite-dimensional closed subspace of $c_{0}(\Gamma)$ has a closed subspace which is isomorphic to $c_{0}$. This clearly implies that $c_{0}(\Gamma)$ contains no closed infinite-dimensional reflexive subspaces. Therefore $J(X)$ is not a closed subspace of $c_{0}(\Gamma)$. On the other hand, the space $c_{0}(\Gamma)$ has an equivalent $C^{\infty}$ smooth norm $g$ ([11], chapter V, theorem 1.5). Then we can define a $C^{\infty}$ smooth norm $\omega$ in $X$ by $\omega(x)=g(J(x))$, and the norm $\omega$ happens to be non-complete because the subspace $J(X)$ is not closed in $c_{0}(\Gamma)$. This concludes the proof of lemma 2.3.

Using the properties of the functional $\omega$ we can construct a deleting path as follows. 
Lemma 2.5. Let $(X,\|\cdot\|)$ be a Banach space, and let $\omega$ be a functional satisfying conditions 1, 2, and 5 of lemma 2.3. Then, for every $\delta>0$, there exists a $C^{\infty}$ path $p=p_{\delta}:(0, \infty) \longrightarrow X$ such that

1. $\omega(p(\alpha)-p(\beta)) \leq \frac{1}{2}(\beta-\alpha)$ if $\beta \geq \alpha>0$;

2. For every compact set $K \subset X$ there exists $t_{0}>0$ such that

$$
\inf \left\{\omega(z-p(t)) \mid 0<t \leq t_{0}, z \in K\right\}>0 ;
$$

3. $p(t)=0$ if and only if $t \geq \delta$.

Proof. For a given $\delta>0$, choose a sequence $\left(y_{k}\right)$ satisfying condition 5 of lemma 2.3, and pick a non-increasing $C^{\infty}$ function $\gamma:[0, \infty) \longrightarrow[0,1]$ such that $\gamma=1$ in $[0, \delta / 2], \gamma=0$ in $[\delta, \infty)$, and $\sup \left\{\left|\gamma^{\prime}(t)\right|: t \in[0, \infty)\right\} \leq$ $4 / \delta$. Then define a path $p:(0, \infty) \longrightarrow X$ by

$$
p(t)=\sum_{k=1}^{\infty} \gamma\left(2^{k-1} t\right) y_{k} .
$$

It is clear that $p$ is a well-defined $C^{\infty}$ path. If $\beta \geq \alpha$ then $\gamma\left(2^{k-1} \alpha\right)-$ $\gamma\left(2^{k-1} \beta\right) \geq 0$ because $\gamma$ is non-increasing, and also $\gamma\left(2^{k-1} \alpha\right)-\gamma\left(2^{k-1} \beta\right)$ $\leq \frac{4}{\delta}\left|2^{k-1} \alpha-2^{k-1} \beta\right|$ because $\sup \left\{\left|\gamma^{\prime}(t)\right| \mid t \in[0, \infty)\right\} \leq 4 / \delta$. Taking this into account and using the properties of $\omega$ listed in lemma 2.3, we may estimate as follows

$$
\begin{aligned}
& \omega(p(\alpha)-p(\beta))=\omega\left(\sum_{k=1}^{\infty}\left(\gamma\left(2^{k-1} \alpha\right)-\gamma\left(2^{k-1} \beta\right)\right) y_{k}\right) \\
& \leq \sum_{k=1}^{\infty} \omega\left(\left(\gamma\left(2^{k-1} \alpha\right)-\gamma\left(2^{k-1} \beta\right)\right) y_{k}\right) \\
& =\sum_{k=1}^{\infty}\left(\gamma\left(2^{k-1} \alpha\right)-\gamma\left(2^{k-1} \beta\right)\right) \omega\left(y_{k}\right) \\
& \leq \sum_{k=1}^{\infty} \frac{4}{\delta}\left|2^{k-1} \alpha-2^{k-1} \beta\right| \omega\left(y_{k}\right)=\sum_{k=1}^{\infty} \frac{2^{k+1} \omega\left(y_{k}\right)}{\delta}|\beta-\alpha| \\
& \leq \sum_{k=1}^{\infty} \frac{2^{k+1}}{\delta} \frac{\delta}{4^{k+1}}|\beta-\alpha|=\frac{1}{2}(\beta-\alpha)
\end{aligned}
$$

for every $\beta \geq \alpha$. Hence, the first condition is fulfilled.

Let us see that $p$ also satisfies the second condition. For a compact set $K \subset X$, condition 5 of lemma 2.3 provides us with numbers $\eta>0$, $m_{1} \in \mathbb{N}$ such that $\omega\left(z-\sum_{k=1}^{n} y_{k}\right) \geq 2 \eta$ for all $n \geq m_{1}$ and $z \in K$. Since $\omega\left(y_{k}\right) \leq \delta / 4^{k+1}$ for every $k$, we can find $m_{2} \in \mathbb{N}$ so that $\sum_{k=m_{2}+1}^{\infty} \omega\left(y_{k}\right) \leq$ $\sum_{k=m_{2}+1}^{\infty} \frac{\delta}{4^{k+1}} \leq \eta$. Let $n_{0}=\max \left\{m_{1}, m_{2}\right\}$, and put $t_{0}=\delta / 2^{n_{0}}$. Then, 
taking into account that $\gamma\left(2^{k-1} t\right)=1$ whenever $0<t \leq t_{0}$ and $1 \leq k \leq$ $n_{0}$, we have

$$
\begin{aligned}
& \omega(z-p(t))=\omega\left(z-\sum_{k=1}^{\infty} \gamma\left(2^{k-1} t\right) y_{k}\right) \\
& =\omega\left[\left(z-\sum_{k=1}^{n_{0}} y_{k}\right)-\left(\sum_{k=1}^{\infty} \gamma\left(2^{k-1} t\right) y_{k}-\sum_{k=1}^{n_{0}} y_{k}\right)\right] \\
& \geq \omega\left(z-\sum_{k=1}^{n_{0}} y_{k}\right)-\omega\left(\sum_{k=1}^{\infty} \gamma\left(2^{k-1} t\right) y_{k}-\sum_{k=1}^{n_{0}} y_{k}\right) \\
& =\omega\left(z-\sum_{k=1}^{n_{0}} y_{k}\right)-\omega\left(\sum_{k=n_{0}+1}^{\infty} \gamma\left(2^{k-1} t\right) y_{k}\right) \\
& \geq \omega\left(z-\sum_{k=1}^{n_{0}} y_{k}\right)-\sum_{k=n_{0}+1}^{\infty} \gamma\left(2^{k-1} t\right) \omega\left(y_{k}\right) \\
& \geq \omega\left(z-\sum_{k=1}^{n_{0}} y_{k}\right)-\sum_{k=n_{0}+1}^{\infty} \omega\left(y_{k}\right) \geq \omega\left(z-\sum_{k=1}^{n_{0}} y_{k}\right)-\sum_{k=m_{2}+1}^{\infty} \omega\left(y_{k}\right) \\
& \geq 2 \eta-\eta=\eta>0
\end{aligned}
$$

for every $0<t \leq t_{0}$ and $z \in K$. In particular,

$$
\inf \left\{\omega(z-p(t)) \mid 0<t \leq t_{0}, z \in K\right\} \geq \eta>0 .
$$

So condition 2 of 2.5 is satisfied as well.

Finally, it is easily seen that the fact that $\left\{y_{k} \mid k=1,2, \ldots\right\}$ is a linearly independent set of vectors ensures that $p(t)=0$ if and only if $t \geq \delta$.

The last thing we will need in order to prove theorem 2.1 is to associate each compact set $K \subset X$ with a function $f: X \longrightarrow[0, \infty)$ such that $f$ is $C^{p}$ smooth on $X \backslash K$, it satisfies $f^{-1}(0)=K$, and $f(x)-f(y) \leq \omega(x-y)$ for every $x, y \in X$. The existence of such functions is ensured by the following lemma, which should be compared with Proposition VIII.3.8 of [11]. It should be noted that $f$ cannot in general be chosen to be $C^{p}$ on the whole space $X$, as a recent paper by P. Hájek [20] shows. Indeed, according to Corollary 9 of [20], for an uncountable $\Gamma$ there is no $C^{2}$ smooth function $f: c_{0}(\Gamma) \longrightarrow[0, \infty)$ with $f^{-1}(0)=0$ (though $c_{0}(\Gamma)$ admits a $C^{\infty}$ norm). The idea of our proof of 2.6 is an adaptation of that of the proof of Lemma 1.1 of [13], where the case of a genuine norm $\omega$ was treated, and the corresponding $f$ was called a $C^{p}$ Whitney function for $K$ (with respect to $\omega)$. 
Lemma 2.6. Let $\omega: X \longrightarrow[0, \infty)$ be a continuous functional satisfying properties 1-3 of lemma 2.3, and such that $\omega$ is $C^{p}$ smooth on $X \backslash\{0\}$. Let $K$ be a compact subset of $X$. For $x \in X$, write $d_{K}(x)=\inf \{\omega(x-$ y) $\mid y \in K\}$. Then, for each $\varepsilon>0$ there exists a continuous function $f=f_{\varepsilon}: X \longrightarrow[0, \infty)$ such that

1. $f$ is $C^{p}$ smooth on $X \backslash K$;

2. $f(x)-f(y) \leq \omega(x-y)$ for every $x, y \in X$;

3. $f^{-1}(0)=K$;

4. $\inf \left\{f(x) \mid d_{K}(x) \geq \eta\right\}>0$ for every $\eta>0$;

5. $f$ is constant on the set $\left\{x \in X \mid d_{K}(x) \geq \varepsilon\right\}$.

\section{Proof of lemma 2.6}

First of all let us see that the function $d_{K}$ is continuous and satisfies $d_{K}^{-1}(0)=K$, and $d_{K}(x)-d_{K}(y) \leq \omega(x-y)$ for every $x, y \in X$. Indeed, for every $y \in X$ and for every $\varepsilon>0$ there exists $y_{\varepsilon} \in K$ such that $d_{K}(y)+\varepsilon \geq \omega\left(y-y_{\varepsilon}\right)$. Then

$$
\begin{aligned}
& d_{K}(x)-d_{K}(y)=\inf \{\omega(x-z) \mid z \in K\}-\inf \{\omega(y-z) \mid z \in K\} \\
& \leq \omega\left(x-y_{\varepsilon}\right)-\omega\left(y-y_{\varepsilon}\right)+\varepsilon \leq \omega\left[\left(x-y_{\varepsilon}\right)-\left(y-y_{\varepsilon}\right)\right]+\varepsilon \\
& =\omega(x-y)+\varepsilon,
\end{aligned}
$$

so that we obtain $d_{K}(x)-d_{K}(y) \leq \omega(x-y)$ by letting $\varepsilon$ go to zero. Since $\omega(z) \leq 2\|z\|$ for every $z$, this inequality implies that $d_{K}(x)-d_{K}(y) \leq$ $2\|x-y\|$ for every $x, y \in X$ and hence $\left|d_{K}(x)-d_{K}(y)\right| \leq 2\|x-y\|$ for every $x, y \in X$, that is, $d_{K}$ is Lipschitz and therefore continuous. The same argument shows that $f$ is Lipschitz if only it satisfies condition 2. On the other hand, if $d_{K}(x)=0$ then there exists a sequence $\left(y_{n}\right) \subseteq K$ such that $\lim _{n} \omega\left(x-y_{n}\right)=0$. Since $K$ is compact we may assume that $\left(y_{n}\right)$ converges to some $y \in K$. By the continuity of $\omega$, we have that $\omega(x-y)=0$, which implies that $x=y \in K$. This, together with the obvious fact that $d_{K}(x)=0$ for every $x \in K$, implies that $d_{K}^{-1}(0)=K$.

Now let us define the sets $U_{n}=\left\{x \in X \mid d_{K}(x)<1 / n\right\}$ for each $n \in$ $\mathbb{N}$. These are open sets satisfying $U_{n+1} \subseteq U_{n}$ for each $n$, and $\bigcap_{n=1}^{\infty} U_{n}=K$. Next, for every $x \in X$ and every $r>0$, we define the asymmetric $\omega$-ball $A(x, r)$ by

$$
A(x, r)=\{z \in X \mid \omega(z-x)<r\} .
$$

It should be noted that the sets $U_{n}$ are $\omega$-open, that is, for every $x \in U_{n}$ there exists $r_{x}>0$ such that $A\left(x, r_{x}\right) \subseteq U_{n}$. Indeed, if $x \in U_{n}$, choose $r=\frac{1}{n}-d_{K}(x)>0$. If $\omega(z-x)<r$ then $d_{K}(z)-d_{K}(x) \leq \omega(z-x)<$ $r=\frac{1}{n}-d_{K}(x)$, so that $d_{K}(z)<1 / n$. This means that $A(x, r)$ is contained in $U_{n}^{n}$.

So, for each $n \in \mathbb{N}$ and each $x \in K$ choose $r_{x}^{n}>0$ such that $r_{x}^{n}<\frac{1}{2 n}$ and $A\left(x, r_{x}^{n}\right) \subseteq U_{n}$. Since, for each $n$ we have $K \subset \bigcup_{x \in K} A\left(x, r_{x}^{n}\right)$, the sets 
$A(x, r)$ are open, and $K$ is compact, there exist $x_{j}^{n} \in K, j=1, \ldots, k(n)$, so that

$$
K \subset \bigcup_{j=1}^{k(n)} A\left(x_{j}^{n}, r_{j}^{n}\right),
$$

where $r_{j}^{n}$ stands for $r_{x_{j}^{n}}^{n}$.

Next, let us see that for every $\omega$-ball $A\left(x_{0}, r\right)$ there exists a $C^{p}$ function $g: X \longrightarrow[0,1]$ such that $A\left(x_{0}, r\right)=g^{-1}(0), g=1$ outside $A\left(x_{0}, 2 r\right)$, and $g(x)-g(y) \leq M \omega(x-y)$ for some $M>0$. Let $h: \mathbb{R} \longrightarrow \mathbb{R}$ be a non-decreasing $C^{\infty}$ function such that $h^{-1}(0)=(-\infty, r]$ and $h=1$ on $[2 r, \infty)$. Let $M=\sup \left\{\left|h^{\prime}(t)\right|: t \in \mathbb{R}\right\}$. Define $g: X \longrightarrow[0, \infty)$ by $g(y)=h\left(\omega\left(y-x_{0}\right)\right)$ for every $y \in X$. It is clear that $A\left(x_{0}, r\right)=$ $g^{-1}(0)$ and $g=1$ outside $A\left(x_{0}, 2 r\right)$. If $\omega\left(y-x_{0}\right)-\omega\left(x-x_{0}\right) \geq 0$ then $g(y)=h\left(\omega\left(y-x_{0}\right)\right) \geq h\left(\omega\left(x-x_{0}\right)\right)=g(x)$ because $h$ is non-decreasing, and then $g(x)-g(y) \leq M \omega(x-y)$ trivially holds. If, on the contrary, $\omega\left(x-x_{0}\right)-\omega\left(y-x_{0}\right) \geq 0$ then, taking into account that $\left|h^{\prime}(t)\right| \leq M$, we get

$$
\begin{aligned}
& g(x)-g(y)=h\left(\omega\left(x-x_{0}\right)\right)-h\left(\omega\left(y-x_{0}\right)\right) \\
& \leq M\left|\omega\left(x-x_{0}\right)-\omega\left(y-x_{0}\right)\right|=M\left(\omega\left(x-x_{0}\right)-\omega\left(y-x_{0}\right)\right) \\
& \leq M \omega(x-y) .
\end{aligned}
$$

In either case we obtain $g(x)-g(y) \leq M \omega(x-y)$ for every $x, y \in X$.

So, for each $\omega$-ball $A\left(x_{j}^{n}, r_{j}^{n}\right)$ let us pick a $C^{p}$ function $g_{(n, j)}: X \longrightarrow$ $[0,1]$ such that $A\left(x_{j}^{n}, r_{j}^{n}\right)=g_{(n, j)}^{-1}(0), g_{(n, j)}=1$ outside $A\left(x_{j}^{n}, 2 r_{j}^{n}\right)$, and $g_{(n, j)}(x)-g_{(n, j)}(y) \leq M_{(n, j)} \omega(x-y)$ for every $x, y \in X$ and some $M_{(n, j)} \geq 1$. Let us note that the product of two non-negative bounded functions satisfying an inequality like $g(x)-g(y) \leq M \omega(x-y)$ also satisfies such an inequality (perhaps with a different $M>0$ ). Indeed, if $g_{1}(x)-g_{1}(y) \leq M_{1} \omega(x-y)$ and $g_{2}(x)-g_{2}(y) \leq M_{2} \omega(x-y)$ then

$$
\begin{aligned}
& g_{1}(x) g_{2}(x)-g_{1}(y) g_{2}(y)= \\
& =g_{1}(x) g_{2}(x)-g_{1}(x) g_{2}(y)+g_{1}(x) g_{2}(y)-g_{1}(y) g_{2}(y) \\
& =g_{1}(x)\left[g_{2}(x)-g_{2}(y)\right]+g_{2}(y)\left[g_{1}(x)-g_{1}(y)\right] \\
& \leq g_{1}(x) M_{2} \omega(x-y)+g_{2}(y) M_{1} \omega(x-y) \\
& \leq\left(\left\|g_{1}\right\|_{\infty} M_{2}+\left\|g_{2}\right\|_{\infty} M_{1}\right) \omega(x-y),
\end{aligned}
$$

where $\left\|g_{i}\right\|_{\infty}=\sup \left\{\left|g_{i}(z)\right|: z \in X\right\}$. Now, for each $n$, consider the product

$$
\varphi_{n}(x)=\prod_{j=1}^{k(n)} g_{(n, j)}(x) .
$$


The functions $\varphi_{n}: X \longrightarrow[0,1]$ satisfy $\varphi_{n}(x)-\varphi_{n}(y) \leq M_{n} \omega(x-y)$ for every $x, y \in X$, for some $M_{n} \geq 1$, as well as $\varphi_{n}=0$ on $K$, and $\varphi_{n}(x)=1$ whenever $x \in X \backslash U_{n}$ (indeed, if $d_{K}(x) \geq 1 / n$ then $\omega\left(x-x_{j}^{n}\right) \geq d_{K}(x) \geq$ $1 / n \geq 2 r_{j}^{n}$, so that $g_{(n, j)}(x)=1$ for every $j=1, \ldots, k(n)$, which yields $\left.\varphi_{n}(x)=1\right)$.

Finally, choose $m \in \mathbb{N}$ such that $1 / m<\varepsilon$. For every $k \geq m$ we have $\varphi_{k}(x)=1$ whenever $d_{K}(x) \geq \varepsilon$. Then define $f: X \longrightarrow[0,1]$ by

$$
f(x)=\sum_{k=m}^{\infty} \frac{1}{2^{k} M_{k}} \varphi_{k}(x)
$$

for every $x \in X$.

Note that for every $x \in X \backslash K$ there exist an open neighbourhood $V_{x}$ of $x$ and a positive integer $n_{x} \geq m$ such that $\varphi_{n}(y)=1$ whenever $y \in V_{x}$ and $n \geq n_{x}$. Indeed, for each $x \in X \backslash K$ let $n_{x}$ be such that $1 / n_{x}<d_{K}(x)$ and put $V_{x}=\left\{y \in X \mid d_{K}(y)>1 / n_{x}\right\}$. It is clear that $V_{x}$ is an open neighbourhood of $x$, and for each $y \in V_{x}$ we have $y \in X \backslash U_{n}$ for every $n \geq n_{x}$, so that $\varphi_{n}(y)=1$ whenever $n \geq n_{x}$. Then all but finitely many of the functions $\varphi_{n}$ in the series defining $f$ are constant on a neighbourhood of each point in $X \backslash K$, which clearly implies that $f$ is a $C^{p}$ smooth function on $X \backslash K$. It is also clear that $f^{-1}(0)=K$, and $f(x)-f(y) \leq \omega(x-y)$ for every $x, y \in X$. That is, $f$ satisfies conditions $1-3$ of lemma 2.6. Let us see that $f$ also satisfies conditions 4 and 5. For a given $\eta>0$, take $n_{0} \geq m$ such that $1 / n_{0} \leq \eta$. Then, for every $k \geq n_{0}$, we have that $\varphi_{k}(x)=1$ whenever $d_{K}(x) \geq \eta$, and therefore

$$
\begin{aligned}
& \inf \left\{f(x) \mid d_{K}(x) \geq \eta\right\}=\inf \left\{\sum_{k=m}^{\infty} \frac{1}{2^{k} M_{k}} \varphi_{k}(x) \mid d_{K}(x) \geq \eta\right\} \\
& \geq \inf \left\{\sum_{k=n_{0}}^{\infty} \frac{1}{2^{k} M_{k}} \varphi_{k}(x) \mid d_{K}(x) \geq \eta\right\}=\sum_{k=n_{0}}^{\infty} \frac{1}{2^{k} M_{k}}>0 .
\end{aligned}
$$

So condition 4 is also fulfilled. Moreover, $f$ is constant (equal to $\left.\sum_{k=m}^{\infty} M_{k}^{-1} 2^{-k}\right)$ on the set $\left\{x \in X \mid d_{K}(x) \geq \varepsilon\right\}$. This concludes the proof of lemma 2.6.

With all these tools in our hands we can give a proof of theorem 2.1.

\section{Proof of theorem 2.1}

First of all let us take an asymmetric non-complete norm $\omega$ from lemma 2.3. Associated to this functional $\omega$, and for a fixed $\varepsilon>0$, let us choose a function $f=f_{\varepsilon}$ from lemma 2.6. Assuming $f(x)=\delta>0$ whenever $d_{K}(x) \geq \varepsilon$, select a path $p=p_{\delta}$ from lemma 2.5. With these choices, for 
every $x \in X \backslash K$, define

$$
\psi(x)=x+p(f(x)) .
$$

We will prove that $\psi: X \backslash K \longrightarrow X$ is a $C^{p}$ diffeomorphism. Let $y$ be an arbitrary vector in $X$, and let $F_{y}:(0, \infty) \longrightarrow[0, \infty)$ be defined by $F_{y}(\alpha)=f(y-p(\alpha))$ for $\alpha>0$. Let us see that $F_{y}(\alpha)$ satisfies the conditions of 2.2. We have

$$
\begin{aligned}
F_{y}(\beta)-F_{y}(\alpha) & =f(y-p(\beta))-f(y-p(\alpha)) \\
& \leq \omega((y-p(\beta))-(y-p(\alpha))) \\
& =\omega(p(\alpha)-p(\beta)) \leq \frac{1}{2}(\beta-\alpha)
\end{aligned}
$$

for every $\beta \geq \alpha>0$. Hence, the first condition of 2.2 is fulfilled. Let us check that $F_{y}$ also satisfies the second condition. Since the set $y-K=$ $\{y-z \mid z \in K\}$ is compact, condition 2 of lemma 2.5 gives us a $t_{0}=t_{0}(K)$ such that

$$
\inf \left\{\omega(y-z-p(t)) \mid 0<t \leq t_{0}, z \in K\right\}>0 ;
$$

that is to say, there exists a number $\eta>0$ such that

$$
\omega(y-z-p(t)) \geq 2 \eta>0
$$

for every $0<t \leq t_{0}$ and $z \in K$. Obviously, we may suppose that $t_{0} \leq \eta$. For each $t>0$, choose $x_{t} \in K$ such that $d_{K}(y-p(t)) \geq \omega\left(y-p(t)-x_{t}\right)-t$. Then, for every $t$ with $0<t \leq t_{0}$, we have

$$
\begin{aligned}
& d_{K}(y-p(t)) \geq \omega\left(y-x_{t}-p(t)\right)-t \\
& \geq 2 \eta-t \geq 2 \eta-\eta=\eta>0,
\end{aligned}
$$

that is, $d_{K}(y-p(t)) \geq \eta$ for $0<t \leq t_{0}$. Now recall that

$$
\inf \left\{f(x) \mid d_{K}(x) \geq \eta\right\}>0 ;
$$

this means that there exists some $r>0$ such that $f(x) \geq r$ whenever $d_{K}(x) \geq \eta$. Then, for every $0<t \leq t_{0}$ we have $f(y-p(t)) \geq r>0$ and therefore

$$
\limsup _{t \rightarrow 0^{+}} F_{y}(t)=\limsup _{t \rightarrow 0^{+}} f(y-p(t)) \geq r>0,
$$

so that the second condition is also satisfied.

Hence, applying 2.2, the equation $F_{y}(\alpha)=\alpha$ has a unique solution. This means that for any $y \in X$, a number $\alpha(y)>0$ with the property

$$
f(y-p(\alpha(y)))=\alpha(y)
$$


is uniquely determined. This implies that the mapping

$$
\psi(x)=x+p(f(x))
$$

is one-to-one from $X \backslash K$ onto $X$, whose inverse satisfies

$$
\psi^{-1}(y)=y-p(\alpha(y)) .
$$

Indeed, if $\psi(x)=\psi(z)=y$ then $f(y-p(f(x)))=f(x)$ and also $f(y-$ $p(f(z)))=f(z)$, so that $f(x)=f(z)=\alpha(y)>0$ by the uniqueness of $\alpha(y)$, and therefore $x=y-p(\alpha(y))=z$. Moreover, for each $y \in X$, since $\psi(y-p(\alpha(y)))=y-p(\alpha(y))+p(f(y-p(\alpha(y))))=y-p(\alpha(y))+$ $p(\alpha(y))$, the point $x=y-p(\alpha(y))$ satisfies $\psi(x)=y$, and also $x \in X \backslash K$ (because $f(x)=\alpha(y)>0$ and $f^{-1}(0)=K$ ).

As $f$ is $C^{p}$ smooth on $X \backslash K$ and $p$ is also $C^{p}$ smooth, so is $\psi$. Let us define $\Phi: X \times(0, \infty) \longrightarrow \mathbb{R}$ by

$$
\Phi(y, \alpha)=\alpha-f(y-p(\alpha)) .
$$

Since for any $y \in X$ we have $y-p(\alpha(y)) \notin K$, the mapping $\Phi$ is differentiable on a neighbourhood of each point $\left(y_{0}, \alpha\left(y_{0}\right)\right)$ in $X \times(0, \infty)$. On the other hand, since $F_{y}(\beta)-F_{y}(\alpha) \leq \frac{1}{2}(\beta-\alpha)$ for $\beta \geq \alpha>0$, it is clear that $F_{y}^{\prime}(\alpha) \leq \frac{1}{2}$ for every $\alpha$ on a neighbourhood of $\alpha(y)$, and

$$
\frac{\partial \Phi(y, \alpha)}{\partial \alpha}=1-F_{y}^{\prime}(\alpha) \geq 1-1 / 2>0 .
$$

Thus, using the implicit function theorem (see e.g. [9], p.61) we obtain that the mapping $y \rightarrow \alpha(y)$ is of class $C^{p}$ and therefore $\psi: X \backslash K \longrightarrow X$ is a $C^{p}$ diffeomorphism. Moreover, it is obvious that $\psi(x)=x$ whenever $d_{K}(x) \geq \varepsilon$. So, for every $\varepsilon>0$ we have constructed a $C^{p}$ diffeomorphism $\psi_{\varepsilon}: X \backslash K \longrightarrow X$ such that $\psi_{\varepsilon}$ is the identity outside the set $\{x \in X \mid$ $\left.d_{K}(x) \leq \varepsilon\right\}$. This proves, in particular, the first part of theorem 2.1.

Now let us see that if $K$ is contained in an open $\varrho$-ball $B=\{x \in X$ : $\varrho(x)<r\}$ then there exists a diffeomorphism $\varphi: X \longrightarrow X \backslash K$ such that $\varphi$ is the identity outside $B$. Choose a $C^{p}$ diffeomorphism $G: X \longrightarrow X$ transforming $\{x \in X: \varrho(x) \leq r\}$ onto $\{x \in X \mid \omega(x) \leq r\}$ (such a diffeomorphism actually exists, according to Lemma 2.7 stated just below this proof, see also [15]). Since $G(K)$ is a compact set contained in $\{x \in$ $X \mid \omega(x)<r\}$, it is easy to see that there exists some $\varepsilon>0$ such that $G(K)$ is contained in $\{x \in X \mid \omega(x) \leq r-2 \varepsilon\}$. Indeed, consider the tower of open sets $A_{n}=\left\{x \in X \mid \omega(x)<r-\frac{1}{n}\right\}, n=1,2, \ldots$, whose union is $\{x \in X \mid \omega(x)<r\}$. By the compactness of $G(K)$, there exists $n_{0}$ such that $G(K) \subset A_{n_{0}}$. It is enough to choose $\varepsilon>0$ so that $2 \varepsilon<1 / n_{0}$. For the compact set $G(K)$, we can pick a diffeomorphism $\psi_{\varepsilon}: X \backslash G(K) \longrightarrow X$ such that $\psi_{\varepsilon}$ is the identity outside the set $\left\{x \in X \mid d_{G(K)}(x) \leq \varepsilon\right\}$. 
Note that, as $G(K)$ is contained in $\{x \in X \mid \omega(x) \leq r-2 \varepsilon\}$, the set $\left\{x \in X \mid d_{G(K)}(x) \leq \varepsilon\right\}$ is contained in $\{x \in X \mid \omega(x) \leq r\}$, so that $\psi_{\varepsilon}$ is the identity outside the latter. Then it is quite clear that the function $\varphi_{\varepsilon}: X \longrightarrow X \backslash K$ defined by $\varphi_{\varepsilon}=G^{-1} \circ \psi_{\varepsilon}^{-1} \circ G$ is a $C^{p}$ diffeomorphism between $X$ and $X \backslash K$ satisfying $\varphi_{\varepsilon}(x)=x$ whenever $\varrho(x) \geq r$. This concludes the proof of Theorem 2.1.

Let us formally state the result which we have used in the final part of the preceding proof. First, recall that convex body $U$ (that is, a closed and convex subset with a non-empty interior) in a Banach space $X$ is said to be a $C^{p}$ body provided $U$ is a $C^{p}$ submanifold with one-codimensional boundary $\partial U$. For the sake of simplicity we will assume that $0 \in i n t U$, and we will write $c c U=\{x \in X \mid \forall r>0 \quad r x \in U\}$, which stands for the characteristic cone of $U$. If $U_{1}, U_{2}$ are $C^{p}$ convex bodies in a Banach space $X$, we will say that $U_{1}$ and $U_{2}$ are $C^{p}$ relatively diffeomorphic provided there exists a $C^{p}$ diffeomorphism $\varphi: X \longrightarrow X$ such that $\varphi\left(U_{1}\right)=U_{2}$.

Now we may restate from [15] the following

Lemma 2.7. Let $X$ be a Banach space, and let $U_{1}, U_{2}$ be $C^{p}$ smooth convex bodies such that the origin is an interior point of both $U_{1}$ and $U_{2}$, and $c c U_{1}=c c U_{2}$. Then there exist a $C^{p}$ diffeomorphism $g: X \longrightarrow X$ such that $g\left(U_{1}\right)=U_{2}, g(0)=0$, and $g\left(\partial U_{1}\right)=\partial U_{2}$, where $\partial U_{j}$ stands for the boundary of $U_{j}$.

Recall that a continuous seminorm $\varrho: X \longrightarrow[0, \infty)$ is said to be $C^{p}$ smooth provided it is so away from its set of zeros $F=\varrho^{-1}(0)$. We will finish this section with an observation that appropriate adaptations of the techniques used in the proof of Theorem 2.1 allow us to show that an infinite-codimensional subspace of the form $F=\varrho^{-1}(0)$ is $C^{p}$ smoothly removable from a Banach space $X$ admitting a $C^{p}$ smooth seminorm $\varrho$. This is clearly stated in the following theorem, which is a fundamental ingredient in the proof of the classification of the smooth convex bodies given in the next section.

Theorem 2.8. Let $(X,\|\cdot\|)$ be a Banach space with a $C^{p}$ smooth seminorm $\varrho$ whose set of zeros is a subspace $F$ such that the quotient space $X / F$ is infinite-dimensional. Then, for every $\varepsilon>0$ there exists a $C^{p}$ diffeomorphism $\varphi$ between $X$ and $X \backslash F$ satisfying $\varphi(x)=x$ whenever $\varrho(x) \geq \varepsilon$.

\section{Classification of smooth convex bodies}

Making use of his pioneering results on negligibility, V. L. Klee [22] gave a topological classification of the convex bodies of a Hilbert space. This result was generalized to every Banach space with the help of Bessaga's 
non-complete norm technique (see the book by Bessaga and Perczyński [7]). To get a better insight in the history of the topological classification of convex bodies the reader should also look at the papers by Stocker [25], Corson and Klee [10], and Bessaga and Klee [5,6]. In [15], T. Dobrowolski gave a $C^{p}$ smooth version of that result which held within the class of WCG Banach spaces. The results of the preceding section enable us to eliminate this restriction, yielding a general result on the smooth classification of the smooth convex bodies of every Banach space.

For the terms needed below, please refer to the text preceding Lemma 2.7; here we also assume that $0 \in i n t U$.

Theorem 3.1. Let $U$ be a $C^{p}$ convex body in a Banach space $X$.

(a) If ccU is a linear subspace of finite codimension ( say $X=c c U \oplus Z$, with $Z$ finite-dimensional), then $U$ is $C^{p}$ relatively diffeomorphic to $c c U+$ $\{z \in Z:|z| \leq 1\}$, where $|\cdot|$ is an Euclidean norm in $Z$.

(b) If ccU is not a linear subspace or $c c U$ is a linear subspace such that the quotient space $X / c c U$ is infinite-dimensional, then $U$ is $C^{p}$ relatively diffeomorphic to a closed half-space (that is, $\left\{x \in X \mid x^{*}(x) \geq 0\right\}$, for some $x^{*} \in X^{*}$ ).

Proof: In order to prove this theorem it suffices to repeat the argument of [15], using corollary 2.8 above. Indeed, a careful examination of the proof of the main theorem in [15] reveals that the argument holds for every Banach space provided we are able to show that for every Banach space $X$, every closed linear subspace $F$ such that $X / F$ is infinite-dimensional, every $C^{p}$ smooth seminorm $\varrho: X \longrightarrow[0, \infty)$ such that $\varrho^{-1}(0)=F$, and every $\varepsilon>0$, there exists a $C^{p}$ diffeomorphism $H: X \longrightarrow X \backslash F$ with $H(x)=x$ whenever $\varrho(x) \geq \varepsilon$. This is exactly what theorem 2.8 reads.

Once we know how to delete points or subspaces in spaces having smooth norms or seminorms, it is not difficult to delete smooth convex bodies. One can give a straightforward proof of this fact, but it will be more convenient for us to deduce it from theorem 3.1.

Theorem 3.2. Let $X$ be a Banach space, and let $U$ be a $C^{p}$ smooth convex body such that its characteristic cone is either a linear subspace of infinite codimension in $X$ or it is not a linear subspace of $X$. Then there exists a $C^{p}$ diffeomorphism from $X$ onto $X \backslash U$.

Proof. According to theorem 3.1, there exists a $C^{p}$ self-diffeomorphism of $X$ mapping $U$ onto a closed half-space. Therefore $X \backslash U$ is $C^{p}$ diffeomorphic to an open half-space. Since an open half-space is obviously $C^{\infty}$ diffeomorphic to the whole space, we may conclude that $X \backslash U$ and $X$ are $C^{p}$ diffeomorphic. 


\section{Garay's phenomena for ODE's in Banach spaces}

Perhaps one of the most unexpected applications of negligibility theory is that found by Barnabas M. Garay [16,17] concerning some strange topological properties of cross-sections of solution funnels for ordinary differential equations in infinite-dimensional Banach spaces. Garay made use of negligibility theory to study the geometry of the failure of Kneser and Peano's theorems in infinite-dimensional Banach spaces. He showed that, for several classes of infinite-dimensional Banach spaces, including the separable Hilbert space, every compact set can be represented as a cross-section of a solution funnel to some ordinary differential equation. The results of section 2 enable us to extend Garay's theorems to the class of all Banach spaces having $C^{p}$ smooth norms, with $p \in \mathbb{N} \cup\{\infty\}$. As a matter of fact, combining Garay's results with theorem 2.1, one can obtain the following

Theorem 4.1 (Garay). Let $X$ be an infinite-dimensional Banach space with an equivalent Fréchet differentiable norm $\|\cdot\|$. Let A be either a compact set or a bounded $C^{1}$ smooth convex body in $X$. We may assume that $A$ is contained in the unit ball of $X$. Then, there exists a continuous function $f: X \longrightarrow X$ such that $f^{-1}(0)=A, f(x)=x$ whenever $\|x\| \geq 2$, and such that, for every $\left(t_{0}, x_{0}\right) \in \mathbb{R} \times(X \backslash A)$, the differential equation

$$
x^{\prime}=f(x)
$$

has a unique solution passing through $\left(t_{0}, x_{0}\right)$, and the solution is global and unbounded.

as well as

Theorem 4.2 (Garay). Let $X$ be an infinite-dimensional Banach space having a Fréchet differentiable norm $\|\cdot\|$, and let $A \subset X$ be either a compact set with at least two points or a bounded $C^{1}$ smooth convex body. Then there exists a continuous map $F: \mathbb{R} \times X \longrightarrow X$ such that the Cauchy problem

$$
x^{\prime}(t)=F(t, x), \quad x\left(t_{0}\right)=x_{0}
$$

admits a unique (global) solution through each point $\left(t_{0}, x_{0}\right) \neq(0,0)$, while the solutions through $(0,0)$ are not unique and given by

$$
x(t)=\frac{1}{2}\left(t^{2}+t|t|\right) a, \quad a \in A,
$$

which means that at the time $t=1$ the solutions through $(0,0)$ reach all the points of $A$. 
Theorem 4.2 is a relatively easy consequence of Theorem 4.1 , which in turn is immediately deduced by combining our Theorem 2.1 with Theorem 1 of [16]. Nevertheless we will say a few words about the way one can use Theorem 2.1 to obtain Theorem 4.1.

Outline of the proof of 4.1 .

Let $h$ be a $C^{1}$ diffeomorphism from $X \backslash\{0\}$ onto $X \backslash A$ which satisfies $h(x)=x$ if $\|x\| \geq 2$ ( $h$ does exist thanks to theorems 2.1 and 3.2). Let us consider the family of curves

$$
x(t)=h^{-1}\left(h\left(x_{0}\right) e^{t}\right), \quad t \in \mathbb{R}, \quad x_{0} \in X \backslash\{0\},
$$

which are pairwise disjoint and cover the set $X \backslash A$. They provide the solutions to the differential equation $x^{\prime}=g(x)$, where $g(x)=\left[\left(D h^{-1}\right)\right.$ $(h(x))] h(x)$. Let $f_{1}: X \longrightarrow X$ be the extension of $g$ defined by letting $f_{1}=0$ on the set $A$. Then the differential equation $x^{\prime}=f_{1}(x)$ almost satisfy the assertion of 4.1, except that $f_{1}$ might be discontinuous at the points of $A$. One can correct this flaw by putting $f(x)=\phi(x) f_{1}(x)$, where $\phi: X \longrightarrow[0,1]$ is a continuous function such that $\phi^{-1}(0)=A$ and $\phi(x)=1$ whenever $\|x\| \geq 2$.

Then the equation

$$
x^{\prime}=f(x)
$$

has a unique solution passing through each point $\left(t_{0}, x_{0}\right) \in \mathbb{R} \times(X \backslash A)$, and the solution is global and unbounded, while, if $x$ is a bounded global solution of (2) then there exists a point $a \in A$ such that $x(t)=a$ for every $t \in \mathbb{R}$.

Remark 4.3. If, in 4.1, the space $X$ has an equivalent $C^{p}$ smooth norm and, moreover, for the compactum $A$ there exists a $C^{p}$ smooth real-valued function $\phi$ with $\phi^{-1}(0)=A$, then $f$ may be chosen to be $C^{p-1}$ smooth. As shown in [13], for every compactum $K$ of a separable Banach space $X$ there exists a $C^{\infty}$ smooth function $\phi: X \rightarrow[0,1]$ with $\phi^{-1}(0)=K$. However, for a nonseparable Banach space $X$, even if $X$ admits a $C^{\infty}$ norm, such functions need not exist, as a cited result of Hajek's shows. The referee pointed out to us that it would be interesting to identify the Banach spaces $X$ which admit such functions.

\section{Periodic diffeomorphisms without fixed points in Banach spaces}

V. L. Klee also used his results on negligibility [22] to prove that if $X$ is either a non-reflexive Banach space or an infinite dimensional $L^{p}$ space, there exists a two-periodic homeomorphism $f: X \longrightarrow X$ without fixed points. This was somewhat surprising because, for a finite-dimensional space $X, \mathrm{P}$. A. Smith [24] had proved that every prime-periodic homeomorphism of $X$ 
must have a fixed point. Klee even showed that for the Hilbert space $H$ and for each integer $n \geq 2$ there exists a periodic homeomorphism $f: H \longrightarrow H$ of pure period $n$ that has no fixed points. By using the results of the preceding sections, in many Banach spaces these results can now be sharpened so as to obtain periodic diffeomorphisms of arbitrary period $n$ having no fixed points.

Theorem 5.1. Let $X$ be an infinite-dimensional Banach space having a (not necessarily equivalent) $C^{p}$ smooth norm $\varrho$. Then there exists a two-periodic $C^{p}$ diffeomorphism $f: X \longrightarrow X$ such that $f$ has no fixed points and $f$ transforms the ball $\{x \in X \mid \varrho(x) \leq 1\}$ onto itself.

Proof. From theorem 2.1 we get a $C^{p}$ diffeomorphism $\varphi: X \rightarrow X \backslash\{0\}$ such that $\varphi$ is the identity outside the ball $B=\{x \in X \mid \varrho(x) \leq 1\}$. Put $A(x)=-x$ for every $x \in X$ (note that $A$ is a two-periodic linear isomorphism whose only fixed point is the origin, and $A$ takes the ball $B$ onto itself). Define $f: X \longrightarrow X$ by $f(x)=\varphi^{-1}(A(\varphi(x)))$ for every $x \in X$. Then it is clear that $f$ is the desired diffeomorphism.

An adaptation of this argument, using appropiate rotations instead of the function $A(x)=-x$, allows to show the following generalization of Klee's result [22].

Theorem 5.2. Let $X$ be a Banach space of the form $X=Y \times Z$, where $Z$ is a separable infinite-dimensional space which is isomorphic to its cartesian square. Then, for each integer $n \geq 2$ there exists a $C^{\infty}$ diffeomorphism $f: X \longrightarrow X$ of pure period $n$ such that $f$ has no fixed points.

We will finish this paper with a remark on the Borsuk-Ulam coincidencetype theorem in infinite dimensions. Recall that the Borsuk-Ulam theorem says that, for any mapping $f: S^{n} \rightarrow \mathbb{R}^{n}$ of the $n$-dimensional sphere there exists $x_{0} \in S^{n}$ such that $f\left(\alpha\left(x_{0}\right)\right)=f\left(x_{0}\right)$, where $\alpha(x)=-x$ is the antipodal map of $S^{n}$. If $f$ is real-valued then, as it is readily seen, the conclusion above holds if one replaces $\alpha$ by an arbitrary continuous mapping $T: S^{n} \rightarrow S^{n}$. It is easy to see that the following infinite-dimensional version of the Borsuk-Ulam theorem follows from the classical, finite-dimensional one. For every $n \in \mathbb{N}$, and every mapping $f: S \rightarrow \mathbb{R}^{n}$ of a unit sphere $S$ in an infinite-dimensional normed space $X$, there exists $x \in S$ so that $f(-x)=f(x)$. Ulam [23], Problem 167, asked whether this can be extended so as to obtain $f(T x)=f(x)$ for some $x \in S$, where $T$ is a self-mapping of $S$ and $X$ is a Hilbert space. In a commentary following the statement of Problem 167 in [23], Klee answered this question in the negative by exhibiting a self-diffeomorphism $T$ of $S$ and a smooth real-valued mapping $f: S \rightarrow \mathbb{R}$ so that $f(T x)=f(x)$ for no $x \in S$. Below we show that the 
same is true for all Banach spaces having (not necessarily equivalent) $C^{p}$ smooth or real-analytic norms.

Remark 5.3. Let $X$ be either an infinite-dimensional Banach space with a $C^{p}$ smooth (not necessarily equivalent) norm $\omega$, or a separable (infinitedimensional) Banach space with a real-analytic (not necessarily equivalent) norm $\omega$. Then there exist a $C^{p}$ (resp. real-analytic) self-diffeomorphism $T$ of $S=\{x \in X \mid \omega(x)=1\}$ and a $C^{p}$ smooth (resp. real-analytic) mapping $f: S \rightarrow \mathbb{R}$ so that $f(T x)=f(x)$ for no $x \in S$.

Proof. By [1] (resp. [14]), $S$ is $C^{p}$ (resp. real-analytic) diffeomorphic to a hyperplane $E$ of $X$. Now it suffices to exhibit the required $T$ and $f$ on $E$. This is trivial. Take a continuous linear functional $x^{*}$ on $E$ and a vector $x_{0} \in E$ so that $x^{*}\left(x_{0}\right) \neq 0$, and let $T$ be the shift $T x=x+x_{0}$. Clearly, $x^{*}(T x)=x^{*}(x)$ for no $x$.

Acknowledgements. The authors wish to thank the referee for many helpful suggestions which improved the exposition.

\section{References}

[1] D. Azagra, Diffeomorphisms between spheres and hyperplanes in infinite-dimensional Banach spaces, Studia Math. 125 (2) (1997), 179-186.

[2] C. Bessaga, Every infinite-dimensional Hilbert space is diffeomorphic with its unit sphere, Bull. Acad. Polon. Sci., Sér. Sci. Math. Astr. et Phys. 14 (1966), 27-31.

[3] C. Bessaga, Negligible sets in linear topological spaces, Bull. Acad. Polon. Sci., Sér. Sci. Math. Astr. et Phys. 16 (1968), 117-119.

[4] C. Bessaga, Interplay Between Infinite-Dimensional Topology and Functional Analysis. Mappings Defined by Explicit Formulas and Their Applications, Topology Proceedings, 19 (1994).

[5] C. Bessaga, V. L. Klee, Two topological properties of topological linear spaces, Israel J. Math. 2 (1964), 211-220.

[6] C. Bessaga, V. L. Klee, Every non-normable Fréchet space is homeomorphic with all of its closed convex bodies, Math. Ann. 163 (1966), 161-166.

[7] C. Bessaga, A. Pelczynski, Selected topics in infinite-dimensional topology, Monografie Matematyczne, Warszawa 1975.

[8] D. Burghelea, N. H. Kuiper, Hilbert manifolds, Annals of Math. 90 (1969), 379-417.

[9] H. Cartan, Calcul différentiel, Hermann, Paris 1967.

[10] H. H. Corson, V. L. Klee, Topological classification of convex sets, Proc. Symp. Pure Math. 7, Convexity Amer. Math. Soc., Providence, R. I. (1963), pp. 37-51.

[11] R. Deville, G. Godefroy, V. Zizler, Smoothness and renormings in Banach spaces, vol. 64, Pitman Monographies and Surveys in Pure and Applied Mathematics, 1993.

[12] J. Diestel, Geometry of Banach Spaces -Selected Topics, Lecture Notes in Mathematics, 485, Springer-Verlag, New York 1975.

[13] T. Dobrowolski, Smooth and R-analytic negligibility of subsets and extension of homeomorphism in Banach spaces, Studia Math. 65 (1979), 115-139. 
[14] T. Dobrowolski, Every Infinite-Dimensional Hilbert Space is Real-Analytically Isomorphic with Its Unit Sphere, Journal of Functional Analysis, 134 (1995), 350-362.

[15] T. Dobrowolski, Relative Classification of Smooth Convex Bodies, Bull. Acad. Polon. Sci., Sér. Sci. Math. 25 (1977), 309-312.

[16] B. M. Garay, Cross sections of solution funnels in Banach spaces, Studia Math. 97 (1990), 13-26.

[17] B. M. Garay, Deleting Homeomorphisms and the Failure of Peano's Existence Theorem in Infinite-Dimensional Banach Spaces, Funkcialaj Ekvacioj, 34 (1991), 85-93.

[18] B. M. Garay, Parallelizability in Banach spaces I-II-III, Acta Math. Hungar., Proc Royal Soc. Edinburgh, Proc. Banach Center Sem.

[19] K. Goebel, J. Wośko, Making a hole in the space, Proc. Amer. Math. Soc. 114 (1992), 475-476.

[20] P. Hájek, Smooth functions on $c_{0}$, To appear in Israel J. Math.

[21] R. C. James, Weakly compact sets, Trans. Amer. Math. Soc. 113 (1964), 129-140.

[22] V. L. Klee, Convex bodies and periodic homeomorphisms in Hilbert space, Trans. Amer. Math. Soc. 74 (1953), 10-43.

[23] R. D. Mauldin (Editor), The Scottish Book, Birkhäuser, Boston 1981.

[24] P. A. Smith, Fixed-point theorems for periodic transformations, Amer. J. Math. 63 (1941), 1-8.

[25] J. J. Stoker, Unbounded convex point sets, Amer. J. Math. 62 (1940), 165-179. 